\title{
INCIDENCIAS DEL SISTEMA CETICOS Y ZOTAC EN LA REGIÓN DE TACNA Y SU ENTORNO DE LA GLOBALIZACIÓN ECONOMICA COMERCIAL EN EL PRESENTE SIGLO
}

\author{
Responsable : :CPC Rubén Soto Huanca \\ Miembro $\quad:$ Mgr. CPC Augusto Cahuapaza Morales
}

\begin{abstract}
RESUMEN
El presente trabajo es motivo de análisis en cuanto a sus efectos posibles en el mediano y largo plazo considerando el entorno global. Las variables que se mueven en ese sentido se modifican dia a día porque la situación se ha vuelto vertiginosa, pues las verdades de hace un año ya no son las mismas y en algunos casos son totalmente distintas. De alli que se tenga que hacer varios pronósticos a fin de predecir algo de lo que pudiera pasar. Esta investigación es viable si contamos con varios elementos que ya se han analizado y otros que pueden obtenerse. No va, desde luego, a satisfacer todas las expectativas del ramo, pero en gran medida tiende a establecer una propuesta.
\end{abstract}

\begin{abstract}
Work, of the present is reason for analysis as far as its possible effects in the medium and long term considering the global surroundings. The variables that move in that sense modify day to day because the situation has become vertiginous, because the truths of a year ago no longer are the same ones and in some cases they are totally different from which it must be made several prognoses in order predict something of which could happen. This investigation is viable if it is that we counted on several elements that already have been analyzed and other that can be obtained, are not of course going to satisfy all the expectations with the branch, but to a great extent it tends to establish a proposal.
\end{abstract}

\section{INTRODUCCIÓN}

El presente trabajo de investigación se da como inicio de una preocupación, siendo motivo de análisis en cuanto a sus efectos posibles en el mediano y largo plazo considerando el entorno global.

Las variables que se mueven en ese sentido se modifican dia a dia porque la situación se ha vuelto vertiginosa, pues las verdades de hace un año ya no son las mismas y en algunos casos son totalmente distintas, de alli que se tenga que hacer varios pronósticos a fin de predecir algo de lo que pudiera pasar.

Cabe indicar que la presente investigación es cualitativa y cuantitativa por lo que no ponemos en consideración la determinación cronológica del trabajo de investigación, mas síla determinación geográfica. Para el presente trabajo sólo estamos tomando la ciudad de Tacna y como muestra representativa 28 comerciantes.

\section{1) Objetivo General}

Determinar los potenciales beneficios del sistema CETICOS para Tacna y del establecimiento de la Zona Franca, asi como su viabilidad en el contexto global del presente siglo.

\section{2) Objetivos Específicos}

Determinar los beneficios obtenidos en el sistema CETICOS ZOTAC en la región de Tacna.

Proponer el establecimiento de la Zona Franca y determinar los potenciales beneficios del sistema en la región de Tacna.

\section{MARCOTEÓRICO}

La Zona Franca de Tacna tiene un historial que data del año 1989 cuando el gobierno crea la ZOTAC empezando a operar en el año 1990, La zona de Tratamiento Especial Comercial, o Zona Franca Comercial de Tacna, se creó con la intención de combatir el contrabando y reducir la gran influencia económica que en esta ciudad fronteriza ejercia la vecina ciudad de Arica, estableciendo un régimen aduanero especial para los artículos ingresados por el puerto de llo y el aeropuerto de Tacna, que pagaban un arancel de sólo 10\% en lugar del usual $25 \%$.

Los comerciantes, además, gozarian de la exoneración del impuesto general a las ventas $18 \%$ (hoy $19 \%$ ) para los articulos comercializados dentro de Tacna. La Zotac tuvo tal acogida que, al cumplirse el primer año de su establecimiento, los comerciantes ariqueños ya expresaban su preocupación porque el volumen normal de sus ventas se había reducido en un $50 \%$; asimismo, en Tacna se habia recaudado para entonces más de ocho millones de dólares por concepto del arancel del $10 \%$.

La Zotac no sólo redujo radicalmente el contrabando proveniente del eje Iquique-Arica, sino que generó importantes ingresos aduaneros, con los que se construyó la recientemente terminada carretera costanera Tacna-llo.La ley de creación de la Zotac preveía que este régimen preferencial duraría 15 años; pero, debido a limitaciones en el desarrollo de las actividades industriales,en el año $1995 \mathrm{se}$ cambió la ley de ZOTAC por la de CETICOS Tacna (Centros de Exportación, Transformación, Industria, Comercialización y Servicios) que planteaba el desarrollo de llo-MataraniTacna, y otorgaba a las industrias establecidas en la zona las mismas facilidades que ofrecia la ZOTAC (exoneración del Impuesto a la Renta y Arancel Cero), pero con la condición de 
exportar como mínimo el $90 \%$ de la producción; si el industrial queria introducir parte de su producción en el mercado nacional, deberia pagar todos los impuestos vigentes.

Estos sistemas no tuvieron la repercusión esperada por el pueblo de Tacna, finalmente a partir del mes de diciembre de 2002 entro en vigencia la Ley N ${ }^{0} 27688$ la Zona Franca y Comercial de Tacna ZOFRATACNA, sobre la base de las organizaciones creadas por las dos leyes anteriores, para la realización de actividades industriales, agroindustriales, de maquila y de servicios, y de la zona comercial de Tacna, que tiene como finalidad contribuir al desarrollo socioeconómico sostenible del departamento de Tacna, a través de la promoción de la inversión y desarrollo tecnológico.

Esta reglamentación permite que el turista compre mercaderías por un valor de hasta US $\$ 1000$ por viaje, con un máximo de tres viajes al año.

\section{METODOLOGÍADEINVESTIGACIÓN}

\section{1) Tipo de Investigación: Básico}

\section{2) Muestra del Estudio}

\section{Poblacion y Muestra}

Población. En el presente trabajo se tomó una población de los comerciantes inscritos en el Registro Único de Contribuyentes.

La muestra seleccionada ha sido calculada con un nivel de confianza del $68 \%$; de acuerdo con la fórmula del muestreo se tiene un margen de error de $32 \%$; que de acuerdo con la tabla de estadistica es un nivel óptimo de confianza.La muestra calculada cuantitativamente para observar los beneficios obtenidos en el sistema CETICOS y ZOTAC de la región de Tacna, de acuerdo con la respectiva fórmula de estadisticas, obtendrá un nivel óptimo de confianza.

\section{3) Tesis y Variables}

\section{Hipótesis de Trabajo}

PRIMERA.- Los beneficios obtenidos en el sistema CETICOS en la región de Tacna, tienen un nivel bajo.

SEGUNDA.- El establecimiento de la zona franca en la región de Tacna, los beneficios del sistema es significante.

\section{Variables del Estudio}

\section{Variable Independiente:}

En la primera hipótesis expresada por "Beneficios obtenidos en el sistema CETICOS en la región de Tacna.

En cuanto a la variable independiente de la segunda hipótesis, está representada por "El establecimiento de la zona franca en la región de Tacna".

\section{Variables dependiente:}

En la primera hipótesis de trabajo de la variable dependiente esta expresada por "tienen un nivel bajo".

En la segunda hipótesis la variable dependiente esta representada por "los beneficios del sistema es significante".

\section{RESULTADOS DE LA INVESTIGACIÓN}

\section{1) Descripción del Trabajo de Campo.}

Para el presente trabajo de investigación se realizó mediante una encuesta de tipo cuestionario que posee caracteristicas cualitativas y cuantitativas en el aspecto de los beneficios obtenidos en el sistema CETICOS en la región de Tacna; por lo que se encuestó según la muestra a los siete comerciantes; en los siguientes items daremos a conocer los resultados por cada objetivo y posteriormente la contrastación de la hipótesis planteada.

\section{2) Resultados Obtenidos Referente a los Beneficios Obtenidos en el Sistema CETICOS en la Región de Tacna.}

Para determinar los beneficios obtenidos en el sistema CETICOS en la región de Tacna, se formularón preguntas en la encuesta realizada las que son: los beneficios que obtuvo en el sistema CETICOS tuvo un nivel $A=$ Alto ; B) Medio y $C$ ) Bajo, de los cuales tenemos los resultados en los items siguientes.

Los beneficios obtenidos en el Sistema Ceticos en la region de Tacna.

\section{Cuadro $\mathrm{N}^{\circ}$ 01:}

\begin{tabular}{|c|c|c|}
\hline \multicolumn{3}{|c|}{$\begin{array}{c}\text { BENEFICIOS OBTENIDOS EN EL } \\
\text { SISTEMA CETICOS }\end{array}$} \\
\hline RESPUESTAS & FRECUENCIA & $\%$ \\
\hline A.- NIVELALTO & 1 & 14,29 \\
\hline B.-NIVEL BANO & 6 & 85,71 \\
\hline C. - NIVEL MEDIO & 0 & 0,00 \\
\hline TOTAL & 7 & 100,00 \\
\hline
\end{tabular}

Fuente: Cuadro elaborado según la pregunta 1 del Anexo 1

\section{Gráfica $N^{\circ} 01$ :}

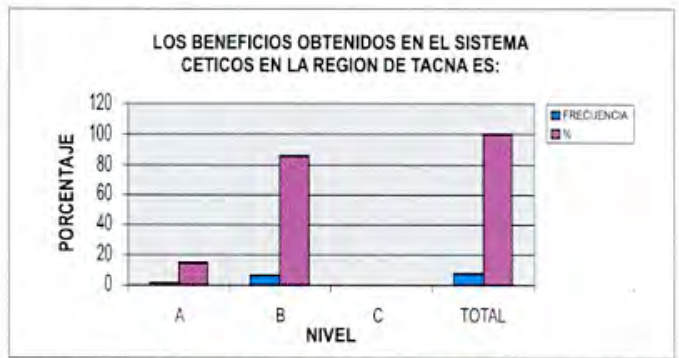

Fuente: Gráfica elaborada en función del Cuadro N01 Donde:

Nivel Alto.

Nivel Bajo.

Nivel Medio 
A través del análisis del Cuadro $\mathrm{N}^{\circ} 01$ y por consiguiente de la Gráfica $N^{0} 01$, podemos decir enfáticamente que en la alternativa ( $A$ ) un comerciante dice que los beneficios obtenidos en el Sistema CETICOS en la región de Tacna tienen un nivel alto, lo que significa porcentualmente el $14,29 \%$; y en la alternativa (B) un número de seis comerciantes optan por decir que los beneficios obtenidos en el Sistema CETICOS en la región de Tacna tienen un nivel bajo lo que significa el $85,71 \% \mathrm{y}$, consecuentemente, ningún comerciante optó por la alternativa (C).

Por lo que queda demostrado que los beneficios obtenidos en el Sistema CETICOS en la región de Tacna tienen un nivel bajo, lo que significa que el sistema no ha tenido acogida por tratarse de la falta de incentivos de parte del Gobierno Central; en cuanto a exoneración para la exportación del sector industrial, ya que se tiene como competidor al vecino pais de Chile que si da exoneración para la exportación del sector industrial.

\section{3) Resultados Obtenidos Referentes al Establecimiento de la Zona Franca en Comparación con el Sistema CETICOS en la Región de Tacna.}

Para determinar el establecimiento de la Zona Franca en comparación del Sistema CETICOS en la región de Tacna, hemos procedido a realizar la encuesta con las siguientes preguntas: Los beneficios del sistema CETICOS en comparación con el establecimiento la Zona Franca es, a) alto; b) igual; c) bajo, de los cuales tenemos los resultados en los items siguientes. Los Beneficios del Sistema Ceticos en Comparación con el Establecimiento de laZona Franca.

\section{Cuadro $\mathrm{N}^{\circ} 02$ :}

\begin{tabular}{|c|c|c|}
\hline \multicolumn{3}{|c|}{ LOS BENEFICIOS DEL SISTEMA CETICOS } \\
\hline EN COMPARACION EST. ZONA FRANCA \\
\hline RESPUESTAS & FRECUENCIA & $\%$ \\
\hline A.-BENEFICIO ALTO & 1 & 14,29 \\
\hline B.-BENEFICIO IGUAL & 1 & 14,29 \\
\hline C.-BENEFICIO BAJO & $\mathbf{5}$ & 71,42 \\
\hline TOTAL & $\mathbf{7}$ & 100,00 \\
\hline
\end{tabular}

Fuente: Cuadro elaborado en base a la pregunta número 2 del anexo.

\section{Gráfica Nº 02}

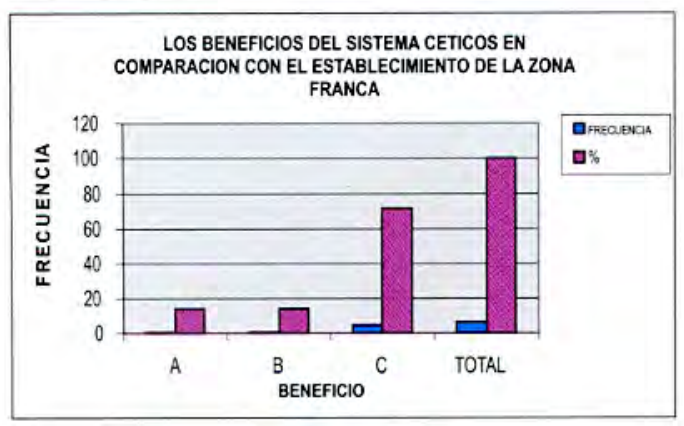

Fuente: Gráfico elaborado según base el Cuadro № 06
Donde:

A. Beneficios Altos.

B. Beneficios Iguales

C. Beneficios Bajos

A través del análisis del Cuadro 2 los beneficios del sistema CETICOS en comparación con el establecimiento la Zona Franca. Por consiguiente, de la Gráfica $N^{\circ} 02$ podemos decir enfáticamente que en la alternativa (A) un comerciante menciona que los beneficios son altos, lo que significa porcentualmente el $14,29 \%$ y en la alternativa (B), un número de un comerciante opta por decir que los beneficios son iguales, lo que significa el $14,29 \%$ y, consecuentemente, en un numero de cinco comerciantes optó por la alternativa (C), lo que significa porcentualmente en un $71,42 \%$. por lo que podemos decir que los beneficios del sistema CETICOS son bajos en comparación con el establecimiento de la Zona Franca.

Por lo que queda demostrado que los beneficios del sistema CETICOS en comparación con el establecimiento la Zona Franca existe una gran diferencia debido a que el mencionado sistema posee una gran diversidad de rubros y partidas arancelarias.

\section{CONCLUSIONES}

1. Los beneficios obtenidos en el sistema CETICOS en la región de Tacna tienen un nivel bajo. Asi tenemos: en la alternativa $(A)$ un comerciante dice que los beneficios obtenidos en el Sistema CETICOS en la región de Tacna tienen un nivel alto, lo que significa porcentualmente el $14,29 \%$ y en la alternativa (B) un número de seis comerciantes optan por decir que los beneficios obtenidos en el Sistema CETICOS en la región de Tacna tienen un nivel bajo lo que significa el $85,71 \%$ y, consecuentemente, ningún comerciante opto por la alternativa $(\mathrm{C})$.

Por lo que queda demostrado que los beneficios obtenidos en el Sistema CETICOS en la región de Tacna tienen un nivel bajo, lo que significa que el sistema no ha tenido acogida por tratarse de la falta de incentivos de parte del Gobierno Central; en cuanto a exoneración para la exportación del sector industrial, ya que se tiene como competidor al vecino pais de Chile que si da exoneración para la exportación del sector industrial.

2. El establecimiento de la Zona Franca en la región de Tacna los beneficios del sistema es significante. Así tenemos: en la alternativa $(A)$ un comerciante menciona que los beneficios son altos, lo que significa porcentualmente el $14,29 \%$ y en la alternativa (B) un número de un comerciante optan por decir que los beneficios son iguales lo que significa el $14,29 \%$ y consecuentemente en un número de cinco comerciantes opto por la alternativa $(C)$, lo que significa porcentualmente en un $71,42 \%$, por lo que podemos decir que los beneficios del sistema CETICOS son bajos en comparación con el establecimiento la Zona Franca.

Por lo que queda demostrado que los beneficios con el establecimiento la Zona Franca es significante debido 
a que el mencionado sistema posee una gran diversidad de rubros y partidas arancelarias.

\section{BIBLIOGRAFÍA}

Ley $N^{\circ} 27688$, Ley de Zona Franca Y Zona Comercial de Tacna, Publicada el 28.03.2002, Y Norma Modificatoria (en Adelante, La Ley).

Reglamento de La Ley de Zona Franca Y Zona Comercial de Tacna, Aprobado mediante Decreto Supremo $N^{0}$ 011-
2002-MINCETUR, Publicado el 17.12.2002 (en adelante, el Reglamento).

La Resolución 53 del Aladi. Concerta Sobre Zona Franca de Tacna.

\section{Leyes}

Leturia Podesta Carlos. "introduccion AlAnálisis, Prevision del Modelo Costo Volumen Rentabilidad" (1998), Lima, @ Editorial Horizonte, Pág. 459.

Franco Falcon Justo. Costos para Toma de Decisiones (1997), Lima. 